\title{
Treating our patients with compassion \& respect: interview with Dr. Zhaolin Lu of the Department of Endocrinology of Peking Union Medical College Hospital
}

Submitted Aug 14, 2017. Accepted for publication Aug 16, 2017.

doi: 10.21037/atm.2017.08.30

View this article at: http://dx.doi.org/10.21037/atm.2017.08.30

\section{Editor's note}

A famous doctor in the Eastern Han Dynasty, to commemorate an old man who had transferred the medical knowledge and technology to him, hung a "drug gourd" outside his pharmacy as a sign of practicing medicine. Since then, "Hanging Gourd to Save Patients" (Xuan Hu Ji Shi) has become a proverb and also a mission for ancient and modern doctors.

Dr. Zhaolin Lu (Figure 1) graduated from the Department of Medicine of Shanghai First Medical College (now Fudan University Shanghai Medical College) in 1963 and has served the Department of Endocrinology of Peking Union Medical College Hospital (PUMCH) for nearly six decades. He has witnessed the development of endocrinology in PUMCH and in China.

$\mathrm{He}$ is often affectionately called "Lu Lao" (highly respected senior with a surname of $\mathrm{Lu}$ ) by younger doctors and nurses in PUMCH.

\section{Introduction}

There is an "Expert Lane" on the second floor of the sixth building of the Old Building of Peking Union Medical College Hospital (PUMCH). When we pushed open an aged gate, we saw the long corridor (the Expert Lane) (Figure 2), and we knew that the masters of offices at both sides of the lane were some of the most prestigious retired experts of PUMCH.

Dr. Lu had already been in the office, waiting for us. "I am glad for this chance to share my experiences in the Department of Endocrinology of PUMCH." smiled Dr. Lu.

There were two books on his desk (Figure 3): one was PUMC Endocrinology \& Metabolism, edited by Dr. Yifan Shi [1928-2013], the first Chinese Academy of Engineering (CAE) academician in endocrinology and the former director of the PUMCH Department of Endocrinology, and

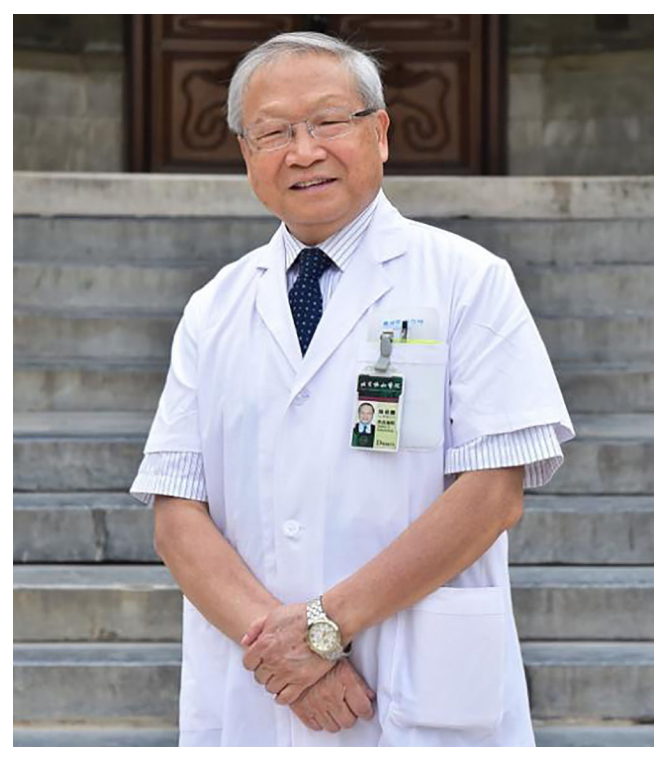

Figure 1 Dr. Zhaolin Lu.

the other was Methods of Hormone Radioimmunoassay, which was brought by him in 1974 when he was trained in the United Kingdom. PUMC Endocrinology \& Metabolism, a classic combines the basic researches and clinical practices in both endocrinology and metabolic disorders, which integrates the general rules with the specific experiences in PUMCH, has long been an absolute must-read book for most endocrinologists in China.

Dr. Lu has studied abroad twice. "Studying abroad was quite different at that time. What you had learned and introduced were urgently needed in China. These new techniques and products not only saved many lives but also directly promoted the development of the whole discipline."

As one of the principal investigators, Dr. Lu participated in the "Clinical and Basic Research on Hormone-secreting Pituitary Tumors", which won the team the national and ministerial scientific and technological progress prizes 
(first grade). Meanwhile, he served as the deputy director and director of PUMCH from 1986 to 1999. "When you were the director of the hospital, were you still involved in clinical work?" We asked. "Of course, a doctor should never leave his patients," answered Dr. Lu.

At the end of the interview, we asked Dr. Lu if he could give young generations of PUMCH Department of Endocrinology any expectations or recommendations. "Of course," said Dr. Lu, by saying in the video that "the spirit

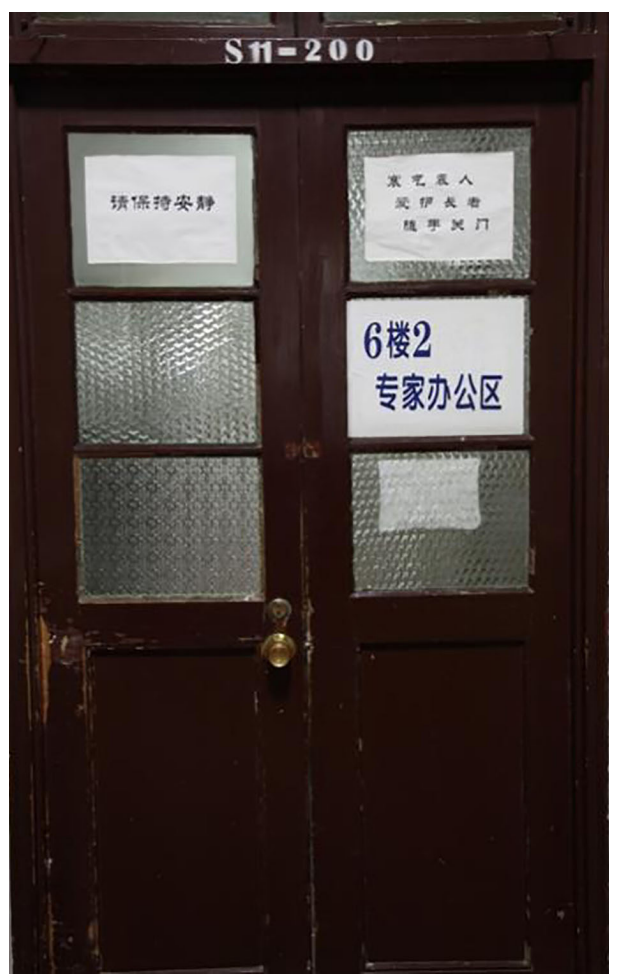

Figure 2 The Expert Lane. of PUMC needs to be inherited and carried forward, which is an unshirkable duty for the younger generations."

Below is a record of Dr. Lu's dictation.

\section{History of PUMCH Department of Endocrinology}

Endocrinology is difficult for many people because it requires a solid biochemical basis. In 1958, Dr. Shihao Liu and his colleagues established China's first department of endocrinology in Peking Union Medical College Hospital, which marked the beginning of endocrinology in China. To promote the development of this discipline, many research teams including pituitary team, diabetic team, thyroid gland team, adrenal gland team, metabolic bone disease team were established in the Department of Endocrinology. Dr. Yifan Shi was responsible for a new brand team-the pituitary team, whereas I was the leader of the adrenal gland team.

Hormone assay is the basis of the clinical practice and research of endocrine disorders. The first thing the pituitary team did was to establish methodology. In early 1960s, the radioimmunoassay was introduced in the western countries. In early 1974, PUMCH sent me to the United Kingdom to learn the radioimmunoassay of hormones. At that time, hormone testing was technically difficult in China because hormone levels are very low in human body and it was almost impossible to measure them with a conventional chemical method. Before the development of radioimmunoassay, Chinese doctors can only rely on clinical manifestations and general laboratory findings to judge endocrine diseases. For example, acromegalic patients often have clinical manifestations such as high cheekbones, prominence of eyebrow, ugly facial features, hypertension, and high blood glucose.
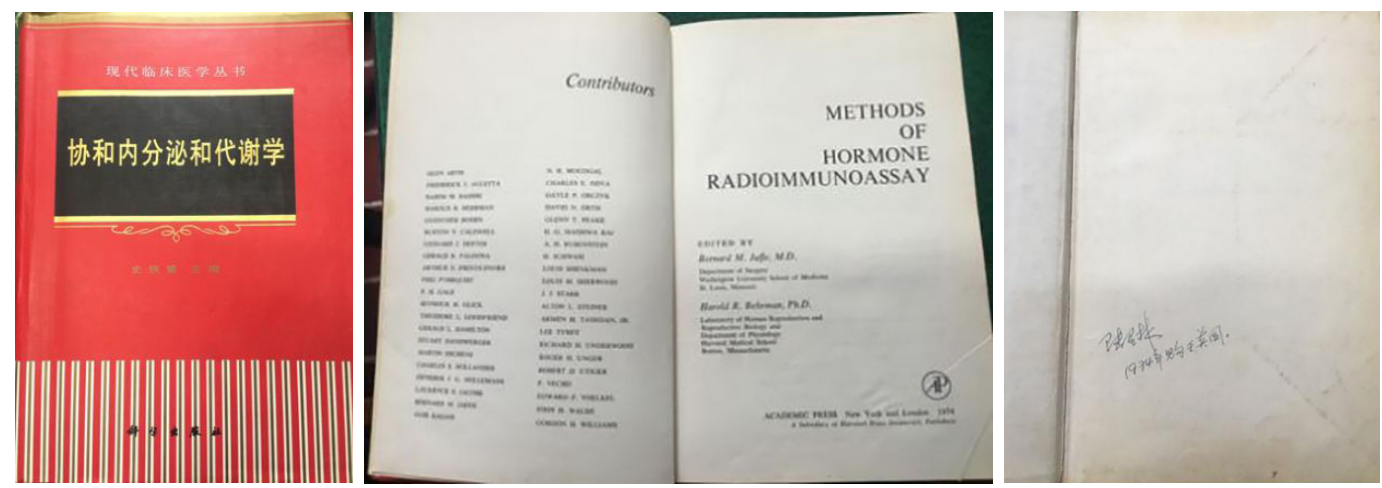

Figure 3 Two books on Dr. Lu's desk-PUMC Endocrinology \& Metabolism and Methods of Hormone Radioimmunoassay. 
Radioimmunoassay combines the sensitivity of radiology with the specificity of immunology and thus can simply and accurately realize the quantitative determination of hormones. With both high sensitivity and high specificity, it was called "revolutionary progress". Its developer, Rosalyn Sussman Yalow, an American immunologist, won the Nobel Prize in Physiology or Medicine in 1977 for development of the radioimmunoassay.

Today radioimmunoassay for measuring hormones is much easier with the commercially available kits. But things were much difficult in 1970s. We had to purify antigens, prepare antibodies, and make labeled compounds by ourselves. After I went to the United Kingdom, I began to learn the whole process of pituitary hormone radioimmunoassay, including antigen preparation (extraction and purification of human pituitary hormones), immunization of animals to produce antibodies with high specificity and high sensitivity, and preparation of highquality iodine-125-labelled antigens. Dr. Michael Bessel from the Department of Endocrinology, St Bartholomew's Hospital, University of London was my mentor. He was very kind and provided me with good learning conditions. I not only learned theories but also took part in every experimental step. When I was planning to return China, my mentor also gave me his lab's high-purity human growth hormone as a gift.

After returning home, I, together with Dr. Jieying Deng, began to develop human growth hormone radioimmunoassay in the Department of Endocrinology. Starting with the preparation of growth hormone antibodies and labeling them by ourselves, we spent more than 1 year to establish the radioimmunoassay method and began to use it in the clinical settings in the second year. This was the first time that the radioimmunoassay of growth hormone was established in China, which laid a solid foundation for the clinical practice and scientific research of pituitary growth hormone tumor in the coming years.

The Department of Endocrinology has a characteristicthe diagnosis, treatment, and research of the endocrine disorders are always based on the laboratory findings. For decades, the PUMCH Department of Endocrinology has long been practicing the feature and essence of "clinical settings-laboratory-clinical settings". PUMCH Department of Endocrinology has its own laboratory, with specialized staff carrying out laboratory research. In addition to hormone testing, the laboratory also carried out many other molecular biology-related works such as gene sequencing and fragment knock-out.

The first step in pituitary tumor research is functional testing, which includes excitation and suppression tests. At present, the normal reference values used in the functional tests by PUMCH are often different from those indicated on some kits. This is because the reference values on these kits are mostly based on the data from foreign human studies, whereas those used in PUMCH are based on the measurements in 100 Chinese normal subjects, and most of these subjects are PUMCH stuff and their family members.

Under the guidance of the PUMC' motto "Preciseness, Perseverance, Diligence, and Devotion", PUMCH Department of Endocrinology was the first center that established the tests for seven pituitary hormones and developed the normal values of 11 functional tests for hypothalamus-pituitary-target glands. These important achievements enabled the quantitative diagnosis of pituitary disease and provided reliable information for clinical diagnosis and treatment.

\section{What makes PUMCH Department of Endocrinology so unique}

For decades, many PUMCH departments that manage pituitary diseases have established close partnerships and accumulated valuable experiences.

For example, when somatostatin analogues were used to treat growth hormone-secreting pituitary tumors, Dr. Yifan Shi's team noticed a sharp rise in the number of gallstone patients, which had not been reported worldwide. After in-depth experimental design and a series of clinical studies, Shi and her colleagues concluded that octreotide could increase the risk of gallstone disease; monitoring of gallbladder function is thus recommended after long-term octreotide use for acromegaly.

In addition, Dr. Yuanxiu Lao from the Department of Ophthalmology who had long been involved in visual study had found that not only large pituitary adenoma will compress the optic chiasm and thus cause temporal hemianopia but also pituitary micro-adenoma will affect the blood supply to optic chiasma and thus lead to hemianopia.

Notably, in recent years PUMCH has taken a lead in research on the diagnosis and differential diagnosis of Cushing's syndrome, with outstanding achievements.

Nearly one third of inpatients in the PUMCH Department of Endocrinology are with Cushing's syndrome, which is unimaginable in other hospitals. While Cushing's 
syndrome is a rare disorder, it has become a common disease in our center. Treatment options can be completely different for the same disease with different etiologies. Therefore, etiological identification is particularly important. Differentiation between adrenocorticotropic hormone (ACTH)-secreting pituitary tumors and ectopic ACTH-secreting syndrome is especially challenging. Sometimes it is difficult to find a tumor even if it is highly suspected of ectopic ACTH-secreting tumors. Thanks to the joint efforts of the Department of Endocrinology and the Radiology Department, the first bilateral inferior petrosal sinus blood sampling plus desmopressin (DDAVP) stimulation test in China was established in PUMCH and has proven to be the best strategy for differentiating these two disorders. In addition, surgical treatment of some pituitary ACTH-secreting micro-adenoma is also a globally challenging issue. After many years of efforts, the Department of Neurosurgery in PUMCH has accumulated rich experience and ranks among the international top neurosurgery centers.

Over the years, the PUMCH Department of Endocrinology has been in strict accordance with the guidelines or consensus to carry out the differential diagnosis of Cushing's syndrome, with each step and every detail being strictly followed. In particular, the whole process of inferior petrosal sinus blood sampling reflects the close collaboration among the Department of Endocrinology, Department of Radiology, laboratory, and many other departments. Meanwhile, the PUMCH Department of Endocrinology also undertakes the postoperative recurrence evaluation for the patients. The postoperative recurrence rate can be assessed by comparing the pre- and post-operative ACTH and cortisol values.

Therefore, although surgery remains the mainstream treatment for Cushing's syndrome, many tasks such as definite diagnosis before operation, judgment of specific causes, determination of lesion site, and evaluation of postoperative recurrence are completed by the Department of Endocrinology. PUMCH has rich experiences in the diagnosis and treatment of such diseases; however, all these tasks can not be completed without the strong cooperation among multiple departments and the adherence to the principle of patient-first.

The pituitary tumor cooperation group, participated by the Department of Endocrinology, Department of Neurosurgery, Department of Radiology, Department of Anesthesiology, and laboratory, are on the right way under the leadership and support of the hospital.

\section{Future of PUMCH Department of Endocrinology}

PUMCH has been famous for its rigorous management, and the policies are even stricter in the Department of Endocrinology. A medical record will be asked to rewrite if there is a single typo on it. Each doctor is required to memorize the abstract when making case report. But, in retrospect, the more stringent the environment, the more it can cultivate a rigorous, realistic, and strong character.

Today, the external environment, the hardware and software of the department has become much better for the younger generation. We had much poorer conditions at that time, and even the stethoscope was made of latex tube, which would be softened in the summer. In addition, an intern must perform the routine fecal, blood, and urine examinations by himself/herself and even closely monitor the follow-up visits. However, even in such difficult conditions, we had never forgotten the thinking and research and kept exploring the unknown realms.

Sometimes, we often sigh that there is too much "helplessness" in medicine. Once upon a time there was a female patient from Jiangxi Province. She got pituitary adenoma before she was 30 years old. Initially she received a surgery in the Department of Neurosurgery. Unfortunately, the patient had increasingly frequent relapse after the operation. Finally, intracranial metastasis of pituitary adenoma occurred, which was confirmed to be pituitary ACTH-secreting carcinoma. Clinically pituitary ACTHsecreting adenoma is extremely rare and pathologically its cellular morphology is generally not different from pituitary adenoma. It can not be diagnosed early and can only be confirmed after metastasis. While medicine has advanced at a breathless pace, there is still much "helplessness" to be explored and addressed by the younger generations.

Every day, there are numerous patients with multiple complicated diseases seeking treatment in PUMCH. It is hoped that the younger generation will be able to carry out in-depth clinical studies with such advantaged conditions, which will be helpful for the development of PUMCH Department of Endocrinology and for the whole discipline in China. When young scholars do internships in a foreign institution, he/she should not just quietly do basic research; rather, he/she should seek any chance to attend outpatient services because many problems are hidden in the clinical settings.

The old generations of PUMCH Department of Endocrinology has made many little-known efforts for the development of the discipline. The younger generations 
should keep inherit and carry forward the spirit of the old generations and make PUMCH Department of Endocrinology one of the top centers in this field both in China and abroad.

\section{Dr. Lu in the eyes of the younger generations}

In the eyes of many young clinicians in PUMCH, Dr. Lu's smile is always bathing them in warmth. In the rounds, young doctors sometimes make mistakes. Unlike the severe criticism from many other senior physicians, Dr. Lu's attitude is much different. "This is a mistake, and try your best not to commit it again, otherwise the patient will suffer again." Although his attitude is moderate, the tone is harsh, which is very impressive for young clinicians.

Nowadays, Dr. Lu only comes to the hospital during grand rounds on Tuesday morning or when there are expert outpatient services. However, whenever he was asked about any problem, he never refuses and always answers as carefully as he can. In addition, what the younger generation needs to learn is that Dr. Lu attends every meeting seriously

Cite this article as: Liao L, Zhang $\mathrm{H}$. Treating our patients with compassion \& respect: interview with Dr. Zhaolin Lu of the Department of Endocrinology of Peking Union Medical College Hospital. Ann Transl Med 2017;5(21):437. doi: 10.21037/atm.2017.08.30 and cautiously. When he serves as the chairman of a conference or even a session, he will always carefully read the $\mathrm{CV}$ and PPT presented by each lecturer in advance. $\mathrm{He}$ urges himself to be well prepared for a specific topic; for this purpose, he often carries out a literature search before a meeting.

These virtues are also the spirits of PUMCH Department of Endocrinology to be learned and inherited by younger generations.

\section{Acknowledgements}

None.

\section{Footnote}

Conflicts of Interest: The authors have no conflicts of interest to declare.

(Science Editors: Lili Liao, Han Zhang, ATM, editor@atmjournal.org) 